\title{
Estudio Anatómico en Relación a los Sitios Comprometidos en la Técnica de Implantes Cigomáticos
}

\author{
Anatomical Study in Relation to the Implicated Sites in the Zygomatic Implants Technique \\ "Dámaso González E. ; **Antonio Sanz R. \& ***Rodrigo Hidalgo
}

GONZÁlEZ, E. D.; SANZ, R. A. \& HIDALGO, H. Estudio anatómico en relación a los sitios comprometidos en la técnica de implantes cigomáticos. Int. J. Morphol., 25(1):133-137, 2007.

RESUMEN: La técnica de implantes cigomáticos fue introducida por Branemark et al. (1997) en la búsqueda de una solución simple para pacientes desdentados totales de maxilares sin reborde residual. La tasa de éxito comunicada por estos autores fue de $96.5 \%$.

La técnica descrita Branemark et al. comienza a nivel de la zona palatina de los $2^{\circ}$ premolares, cruzando el reborde alveolar hacia el seno maxilar y a través de éste hacia el borde posterosuperior del hueso cigomático (ángulo yugal), logrando la estabilización del implante a ese nivel.

La presente investigación se realizó en cráneos completos del Departamento de Anatomía Humana de la Facultad de Odontología de la Universidad Mayor, en los cuales se efectuaron mediciones bilaterales de las diferentes estructuras óseas comprometidas en el recorrido del implante cigomático, con el propósito de determinar el recorrido intraóseo de este tipo de implante y su relación con las estructuras que atraviesa.

Independiente del tipo de cráneo analizado, la relación de contacto hueso- implante en el recorrido de éste, es de aproximadamente un tercio de su longitud y así se tiene que en distancias promedio de $40 \mathrm{~mm}$ de recorrido, no más de 10 a $12 \mathrm{~mm}$ de tejido óseo están en contacto con el implante.

PAlabras ClaVE: Huesos cigomáticos; Cara infratemporal de la maxila; Proceso piramidal.

\section{INTRODUCCIÓN}

Los implantes oseointegrados de titanio han demostrado ser un adecuado tipo de tratamiento para desdentados totales, así como para desdentados parciales (Branemark et al., 1997).

Los implantes cigomáticos fueron introducidos por Branemark y su grupo para restaurar la función en pacientes con maxilas severamente reabsorbidas, en las cuales no era posible utilizar implantes en forma convencional. La utilización de esta técnica fue propuesta además para evitar procedimientos más complejos como son los injertos óseos. Los implantes cigomáticos comenzaron a ser utilizados por Branemark y colaboradores en 1989, tiempo desde el cual comenzó el seguimiento de ellos para su análisis estadístico. Este grupo ha reportado el resultado de 81 pacientes tratados con fijaciones en la región cigomática, incluyendo 24 maxilectomías y 3 defectos de fisuras palatinas (Darle, 2000a). El rango de éxito total es de 96,8\% y la reducción en la necesidad de realizar injertos en estos pacientes se estima en un $75 \%$.

El implante cigomático es de titanio maquinado con forma de tornillo de $3.75 \mathrm{~mm}$ de diámetro en la zona media y apical, en su parte inferior, que queda en relación a la cortical alveolar, es de $4.0 \mathrm{~mm}$ de diámetro; su largo va desde 35 $\mathrm{mm}$ a $55 \mathrm{~mm}$ y tiene su plataforma protésica angulada respecto del eje mayor del implante en $45^{\circ}$ para compensar la inclinación de éste (Bedrossian \& Stumpel, 2001).

\footnotetext{
* Profesor Titular Depto. de Anatomía Humana Facultad de Odontología Universidad Mayor, Chile.

** Profesor Adjunto de Implantología Oral Facultad de Odontología Universidad de Chile. Coordinador Unidad de Implantología Curso de Post Grado, Periodoncia e Implantes Universidad Mayor, Chile.

****Especialista en Periodoncia, Universidad de Chile.
} 
El procedimiento clínico para la instalación de implantes cigomáticos se realiza confeccionando un lecho quirúrgico, que va desde la región palatina de los premolares (entre $1^{\circ}$ y $2^{\circ}$ ó a nivel del $2^{\circ}$ ) hasta el borde pósterosuperior del hueso cigomático (ángulo yugal)(Darle, 2000b).

La preparación del lecho quirúrgico se efectúa con fresas de acero en forma ascendente hasta labrar el lecho correspondiente para recibir el implante de $3.75 \mathrm{~mm}$ de diámetro.

La preparación del lecho quirúrgico atraviesa el seno maxilar, para lo cual se labra una ventana en la pared lateral de este seno, lo más alta posible y en relación al proceso cigomático del maxilar, para observar el paso de la fresa. La mucosa del seno maxilar debe ser rechazada para no comprometerla.

Posteriormente, la fresa penetra la pared pósterosuperior del seno maxilar, entrando en la fosa infratemporal y alcanzando el borde pósterosuperior del hueso cigomático (Darle, 2000 a,b; Uchida et al., 2001).

El presente estudio registra las dimensiones de las estructuras óseas y espacios comprometidos en la técnica de implantes cigomáticos antes descrita.

\section{MATERIAL Y MÉTODO}

Fueron analizados 25 cráneos del Departamento de Anatomía Humana de la Facultad de Odontología de la Universidad Mayor, Santiago, Chile.

En cada cráneo se realizaron mediciones bilaterales,

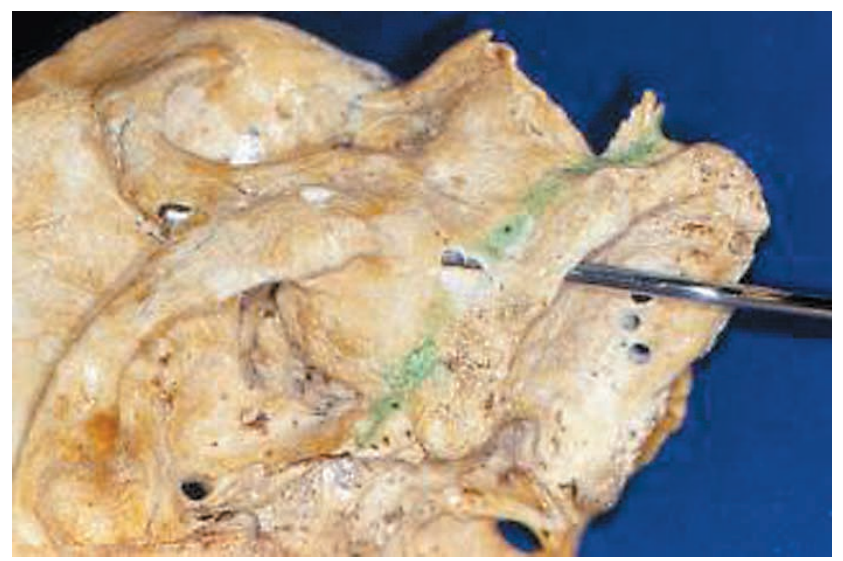

Fig. 1. Sonda colocada en el lecho óseo preparado para el recorrido de un implante cigomático, desde la bóveda del paladar al ángulo pósterosuperior del hueso cigomático (ángulo yugal). para establecer un promedio de las distancias comprometidas en el paso del implante cigomático hacia el ángulo yugal del maxilar, de tal manera de poder estimar el porcentaje de recorrido del implante dentro del tejido óseo.

Las mediciones fueron, con un estilete que atravesó toda la preparación hecha en el cráneo, siguiendo la orientación descrita en la técnica original de Branemark. Fig. 1.

Siguiendo la descripción de la orientación y de las estructuras atravesadas en la preparación del lecho quirúrgico para la instalación del implante cigomático, se procedió a realizar esta misma preparación en cada uno de los 25 cráneos en estudio (Fig. 2). Luego se procedió a medir con un estilete el recorrido en el hueso y en espacios aéreos dentro de la cara y del cráneo, lo que se transportó a través de un compás a una regla graduada milimétricamente. Las anotaciones se recogieron en un cuadro específicamente diseñado para cada zona del cráneo a ser medido e identificada con el número de cráneo en análisis.

Las mediciones realizadas y recogidas en los diferentes cuadros fueron las siguientes:

1. Distancia desde el reborde alveolar a la altura del segundo premolar por su cara palatina al ángulo yugal del hueso cigomático.

2. Distancia desde el reborde alveolar a nivel del $2^{\circ}$ premolar por palatino al punto medio del hueso cigomático.

3. Espesor del hueso cigomático a nivel del borde pósterosuperior (ángulo yugal).

4. Espesor del hueso cigomático en su porción media.

5. Espesor del hueso cigomático a nivel de su borde pósteroinferior.

6. Mediciones promedio en los cráneos de las estructuras relacionadas con el recorrido del implante cigomático.

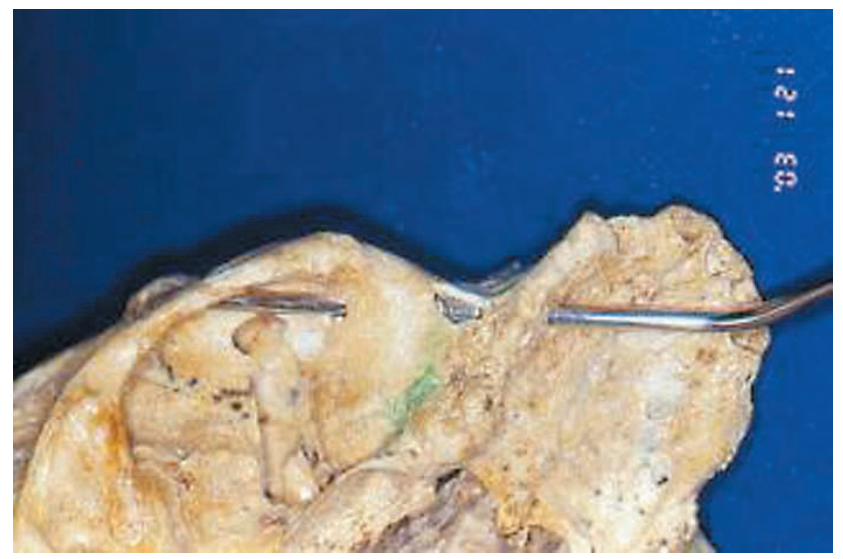

Fig. 2. Sonda en el recorrido del implante cigomático a nivel del seno maxilar y fosa infratemporal, antes de alcanzar ángulo pósterosuperior del hueso cigomático (ángulo yugal). 


\section{RESULTADOS}

Las mediciones propuestas arrojaron los siguientes resultados:

Tabla I. Distancia reborde alveolar nivel $2^{\circ}$ premolar por palatino al ángulo pósterosuperior del hueso cigomático (ángulo yugal o punto de unión entre los procesos frontal y temporal del hueso cigomático). (mm).

\begin{tabular}{|c|c|c|c|c|c|}
\hline $\begin{array}{c}\text { Cráneo } \\
\mathrm{N}^{\circ}\end{array}$ & $\begin{array}{l}\text { Der. } \\
\mathrm{mm}\end{array}$ & $\begin{array}{l}\text { Izq. } \\
\mathrm{mm}\end{array}$ & $\begin{array}{c}\text { Cráneo } \\
\mathrm{N}^{\circ}\end{array}$ & $\begin{array}{l}\text { Der. } \\
\mathrm{mm}\end{array}$ & $\begin{array}{l}\text { Izq. } \\
\text { mm }\end{array}$ \\
\hline 1 & 52 & 52 & 1 & 41 & 41 \\
\hline 2 & 56 & 55 & 2 & 44 & 45 \\
\hline 3 & 59 & 60 & 3 & 42 & 42 \\
\hline 4 & 54 & 54 & 4 & 44 & 43 \\
\hline 5 & 57 & 56 & 5 & 46 & 46 \\
\hline 6 & 51 & 53 & 6 & 42 & 41 \\
\hline 7 & 58 & 57 & 7 & 41 & 41 \\
\hline 8 & 57 & 57 & 8 & 46 & 47 \\
\hline 9 & 49 & 49 & 9 & 41 & 41 \\
\hline 10 & 57 & 57 & 10 & 41 & 41 \\
\hline 11 & 60 & 57 & 11 & 47 & 47 \\
\hline 12 & 56 & 57 & 12 & 49 & 49 \\
\hline 13 & 56 & 56 & 13 & 43 & 42 \\
\hline 14 & 57 & 56 & 14 & 49 & 49 \\
\hline 15 & 51 & 51 & 15 & 45 & 45 \\
\hline 16 & 53 & 52 & 16 & 47 & 46 \\
\hline 17 & 57 & 56 & 17 & 43 & 43 \\
\hline 18 & 51 & 51 & 18 & 48 & 49 \\
\hline 19 & 51 & 51 & 19 & 43 & 41 \\
\hline 20 & 58 & 51 & 20 & 51 & 51 \\
\hline 21 & 49 & 50 & 21 & 49 & 49 \\
\hline 22 & 49 & 51 & 22 & 44 & 44 \\
\hline 23 & 53 & 53 & 23 & 47 & 46 \\
\hline 24 & 54 & 54 & 24 & 50 & 49 \\
\hline 25 & 54 & 54 & 25 & 40 & 40 \\
\hline Media & $54,4 \mathrm{~mm}$ & $54 \mathrm{~mm}$ & Media & $44,9 \mathrm{~mm}$ & $44,7 \mathrm{~mm}$ \\
\hline
\end{tabular}

Tabla III. Espesor hueso cigomático a nivel del ángulo posterosuperior (ángulo yugal). (mm).
Tabla IV. Espesor hueso cigomático en zona media (mm).
Tabla V. Espesor hueso cigomático a aivel de su borde posteroinferior $(\mathrm{mm})$.

\begin{tabular}{ccc}
\hline $\begin{array}{c}\text { Cráneo } \\
\mathrm{N}^{\circ}\end{array}$ & $\begin{array}{c}\text { Der. } \\
\mathrm{mm}\end{array}$ & $\begin{array}{c}\text { Izq. } \\
\mathrm{mm}\end{array}$ \\
\hline 1 & 2,8 & 2,9
\end{tabular}

\begin{tabular}{ccc}
\hline $\begin{array}{c}\text { Cráneo } \\
\mathrm{N}^{\circ}\end{array}$ & $\begin{array}{c}\text { Der. } \\
\mathrm{mm}\end{array}$ & $\begin{array}{c}\text { Izq. } \\
\mathrm{mm}\end{array}$ \\
\hline 1 & 4,5 & 4,5
\end{tabular}

$2 \quad 2,4 \quad 2,4$

$3 \quad 2,4 \quad 2,5$

$4 \quad 2,8 \quad 2,5$

$5 \quad 2,4 \quad 2,3$

$6 \quad 3,3 \quad 3,0$

$7 \quad 3,2 \quad 3,4$

$8 \quad 3,1 \quad 3,0$

$9 \quad 2,6 \quad 1,9$

$10 \quad 3,6 \quad 3,9$

$11 \quad 2,1 \quad 2,1$

$12 \quad 4,2 \quad 4,5$

$13 \quad 3,4 \quad 3,4$

$14 \quad 4,5 \quad 3,9$

$15 \quad 3,9 \quad 3,8$

$16 \quad 3,0 \quad 3,0$

$17 \quad 3,5 \quad 3,3$

$18 \quad 3,5 \quad 3,0$

$19 \quad 2,5 \quad 1,9$

$20 \quad 3,8 \quad 3,6$

$21 \quad 3,0 \quad 3,0$

$22 \quad 2,9 \quad 2,6$

$23 \quad 3,5 \quad 3,8$

$24 \quad 4,3 \quad 4,6$

$\begin{array}{lll}25 & 2,9 & 2,0\end{array}$

Media $3,18 \mathrm{~mm} \quad 3,05 \mathrm{~mm}$
$2 \quad 5,0 \quad 5,7$

$3 \quad 4,6 \quad 4,8$

$4 \quad 5,2 \quad 5,3$

$5 \quad 4,5 \quad 4,6$

$\begin{array}{lll}6 & 5,6 & 5,8\end{array}$

$\begin{array}{lll}7 & 5,9 & 5,5\end{array}$

$\begin{array}{lll}8 & 5,9 & 5,8\end{array}$

$\begin{array}{lll}9 & 5,2 & 4,9\end{array}$

$10 \quad 6,3 \quad 6,4$

$11 \quad 2,8 \quad 2,5$

$128,0 \quad 8,0$

$13 \quad 5,8 \quad 5,3$

$14 \quad 6,5 \quad 6,5$

15

6,0

6,1

16

6,0

6,1

17

5,8

18

4,6

19

5,2

20

4 ,

21

$$
5,
$$

22

5,8

23

5,

$24 \quad 7,5 \quad 8,0$

$25 \quad 3,3 \quad 3,5$

Media $\quad 5,38 \mathrm{~mm} \quad 5,34 \mathrm{~mm}$

\begin{tabular}{|c|c|c|}
\hline $\begin{array}{c}\text { Cráneo } \\
\mathrm{N}^{\circ}\end{array}$ & $\begin{array}{l}\text { Der. } \\
\mathrm{mm}\end{array}$ & $\begin{array}{l}\text { Izq. } \\
\mathrm{mm}\end{array}$ \\
\hline 1 & 2,8 & 3,7 \\
\hline 2 & 3,9 & 4,0 \\
\hline 3 & 5,7 & 6,0 \\
\hline 4 & 4,3 & 5,0 \\
\hline 5 & 4,0 & 4,2 \\
\hline 6 & 4,0 & 4,8 \\
\hline 7 & 5,2 & 5,0 \\
\hline 8 & 4,7 & 7,3 \\
\hline 9 & 4,4 & 5,0 \\
\hline 10 & 6,2 & 6,7 \\
\hline 11 & 5,6 & 5,4 \\
\hline 12 & 7,9 & 7,6 \\
\hline 13 & 5,8 & 5,0 \\
\hline 14 & 5,6 & 6,7 \\
\hline 15 & 6,2 & 6,0 \\
\hline 16 & 4,9 & 4,5 \\
\hline 17 & 6,0 & 6,0 \\
\hline 18 & 6,2 & 6,2 \\
\hline 19 & 5,5 & 5,8 \\
\hline 20 & 4,2 & 4,0 \\
\hline 21 & 5,4 & 6,0 \\
\hline 22 & 4,4 & 4,0 \\
\hline 23 & 5,2 & 5,3 \\
\hline 24 & 5,5 & 5,8 \\
\hline 25 & 6,0 & 5,5 \\
\hline edia & $5,18 \mathrm{~mm}$ & $5,42 \mathrm{mn}$ \\
\hline
\end{tabular}


Tabla VI. Mediciones promedio en cráneos largo fresa desde $2^{\circ}$ premolar por palatino al ángulo yugal.

\begin{tabular}{lc}
\hline Largo fresa desde $2^{\circ}$ premolar por palatino al ángulo yugal. & $54 \mathrm{~mm}$ \\
Largo fresa dentro de la cavidad del seno maxilar. & $24 \mathrm{~mm}$ \\
Largo fresa quirúrgica en la fosa infratemporal. & $18 \mathrm{~mm}$ \\
Largo fresa quirúrgica en el interior del hueso cigomático. & $4 \mathrm{~mm}$ \\
Largo fresa quirúrgica en el reborde residual. & $8 \mathrm{~mm}$ \\
\hline
\end{tabular}

\section{DISCUSIÓN}

Las observaciones realizadas en el estudio de la técnica de implantes cigomáticos en los 25 cráneos analizados, hacen surgir algunas interrogantes que deben ser planteadas.

La técnica descrita por Branemark y colaboradores plantea que el anclaje superior del implante es a nivel del ángulo yugal en la unión del proceso frontal con el proceso cigomático del hueso; sin embargo, las mediciones realizadas demuestran que, en promedio el espesor óseo de este ángulo no supera los 3,18 mm (2.1-4.6) estando la media por debajo de los $3 \mathrm{~mm}$ de grosor. En consecuencia, un implante cuyo diámetro en milímetros a la altura de su zona apical es de $3,75 \mathrm{~mm}$, queda literalmente fuera del hueso en su punto de anclaje superior, generando entonces una interrogante respecto al real valor de este anclaje.

La oseointegración de un implante cigomático se alcanza a nivel del paso de éste por el hueso alveolar y a nivel de su entrada en el hueso cigomático.

Triplett et al. (2000), han reportado que el diámetro de los implantes cigomáticos instalados en el hueso cigomático es de $3,75 \mathrm{~mm}$.

Frodel et al. (1993), han reportado que el hueso que debe rodear a un implante oseointegrado debe ser de al menos $1 \mathrm{~mm}$ de espesor, para conseguir adecuada óseo integración.

Uchida et al., plantearon que el espesor del hueso cigomático en su ángulo yugal debería medir al menos 5,75 $\mathrm{mm}$ de espesor, para poder instalar un implante cigomático.

Por otra parte, Nkenke et al. (2003) plantearon en una evaluación de las características óseas del hueso cigomático, que éste es predominantemente hueso trabecular, haciéndolo poco favorable para la instalación de implantes. Sin embargo, estos mismos autores aseguran que el éxito de los implantes cigomáticos está dado porque en su recorrido atraviesa al menos 4 porciones corticales.
Por otra parte, la investigación realizada revela que el recorrido real del implante dentro de hueso cigomático no supera el $25 \%$ de la distancia recorrida, $12 \mathrm{~mm}$ de $54 \mathrm{~mm}$ del recorrido total. Esto está de acuerdo por lo planteado por Uchida et al. quienes establecen que existe una fuerte correlación entre la altura de los pacientes y la cantidad de recorrido del implante dentro de hueso desde el ángulo superior del seno maxilar al ángulo yugal, a mayor altura más larga la distancia, en individuos bajos más corta la distancia.

Esto puede ser relacionado con publicaciones previas que han informado sobre un fracaso mayor de implantes oseointegrados menores de $10 \mathrm{~mm}$ de largo.

De nuestro estudio es posible extractar las siguientes conclusiones, teniendo en consideración lo reducido de la muestra analizada:

No existen diferencias significativas en las mediciones efectuadas en cráneos y siguiendo la técnica descrita, entre los lados derecho e izquierdo.

El hueso cigomático posee su menor espesor a nivel de su borde pósterosuperior, donde se encuentra el ángulo yugal y que alcanza en promedio a sólo $3,1 \mathrm{~mm}$.

El mayor espesor del hueso cigomático se encuentra en la zona media del hueso y en su borde inferior, en el lugar que se articula con el proceso cigomático del maxilar, alcanzando su grosor promedio de 5,36 $\mathrm{mm}$ y de 5,30 $\mathrm{mm}$, respectivamente.

La confección de un lecho quirúrgico para colocar implantes cigomáticos de 3,75 mm de diámetro, indica que estos implantes no deberían llegar hasta el ángulo yugal (borde pósterosuperior del hueso cigomático), pues en esa zona el grosor del hueso es de tan sólo $3,1 \mathrm{~mm}$ y por lo tanto estos implantes quedan fuera del tejido óseo y en plena fosa infratemporal. 
GONZÁLEZ, E. D.; SANZ, R.A. \& HIDALGO, H. Anatomical study in relation to the implicated sites in the zygomatic implants. Int. J. Morphol., 25(1):133-137, 2007.

SUMMARY: The technique of zygomatic implants introduced by Branemark et al. (1997) in search of a simple solution for total maxillary toothless patients without a residual margin. The rate of success communicated by these authors was of $96.5 \%$.

The technique Branemark et al. describes, begins at the level of the palatine area of the $2^{\circ}$ premolars crossing the alveolar margin toward the maxillary sinus and throughout the same toward the posterosuperior margin, obtaining the stabilization of the implant at that level.

The present investigation was realized in complete cranium of the Department of Human Anatomy of the Dentistry Department of the Universidad Mayor, Santiago, Chile, during which bilateral measurements of the different bone structures affected in the zygomatic implant, with the purpose of determining the intra bone trajectory in this type of implant and its relation with the structures that it crosses.

Independently of the type of cranium analyzed, the relation of the bone-implant contact in itstrajectory, is approximately one third of its longitude and it is determined that in an average distance of $40 \mathrm{~mm}$ or trajectory, no more than 10 to $12 \mathrm{~mm}$ of bone tissue is in contact with the implant.

\section{KEY WORDS: Zigomatic bones; Infratemporal surface of maxilla; Piramidal process.}

\section{REFERENCIAS BIBLIOGRÁFICAS}

Branemark, P. I.; Hansson, B. O.; Adell. R.; Breine, U.; Lindstrom, J.; Hallén, O. \& Öhman, A. Oseeointegrated implants in the treatment of edentulous jaw. Experience from a 10 years period. Scand. J. Plast. Reconstr. Surg. Hand. Surg., 16 (Supl. 1), 1997.

Bedrossian, E. \& Stumpel, L. J. Immediate stabilization at stage II of zigomatic implants: Rationale and technique. J. Prosth. Dent., 86:10, 2001.

Darle, C. A new procedure for rehabilitating the severely resorbed maxilla. Prosthodontic Insigths Newsletter 13(1), 2000a.

Darle, C. The Zygoma Option. $2^{\circ}$ edition. Updated October Nobel Biocare, 2000b.

Frodel, J. L.; Frunk, G. F.; Capper, D. T. et al. Osseointegrated Implants : A comparative study of bone thickness in four vascularized bone flaps. J. plast. Reconstrc. Surg., 192: 449, 1993.

Nkenke, E. ; Hahn; Lell, M.; Wiltfang, J. et al. Anatomic site evaluation of the zygomatic bone for dental implant placement. Clin. Oral. Impl. Res., 14:72-9, 2003.

Triplett, R. G.; Schow, S. R.; Laskin, D. M.Oral and maxillofacial surgery advances in implant dentistry. Int. J. Oral and Maxillofac. Implants, 15:47, 2000.
Uchida, Y. ; Masaaki, G. ; Takeshi, K. \& Toshio, A. Mesurements of the maxilla and zigoma as an aid in installing zygomatic implants. J. Oral. Maxillofac. surgery, 59:1193-8, 2001.

\author{
Dirección para correspondencia: \\ Prof. Dr. Dámaso González \\ Depto. de Anatomía Humana \\ Facultad de Odontología \\ Universidad Mayor \\ Santiago - CHILE
}

Recibido: 27-01-2006

Aceptado:18-11-2006 